\title{
Evidence of thermal conduction suppression in hot coronal loops: supplementary results
}

\author{
Tongjiang Wang ${ }^{1,2}$, Leon Ofman ${ }^{1,2}$, Xudong Sun ${ }^{3}$, Elena \\ Provornikova $^{4}$ and Joseph M. Davila ${ }^{2}$
}

${ }^{1}$ Dept. of Physics, Catholic University of America, 620 Michigan Avenue NE, Washington, DC 20064, USA; email: tongjiang.wang@nasa.gov

${ }^{2}$ NASA Goddard Space Flight Center, Code 671, Greenbelt, MD 20770, USA

${ }^{3}$ W. W. Hansen Experimental Physics Laboratory, Stanford University, Stanford, CA 94305, USA

${ }^{4}$ Space Science Division, Naval Research Laboratory, Washington, DC 20375, USA

\begin{abstract}
Slow magnetoacoustic waves were first detected in hot ( $>6 \mathrm{MK}$ ) flare loops by the SOHO/SUMER spectrometer as Doppler shift oscillations in Fe XIX and Fe XXI lines. Recently, such longitudinal waves have been found by SDO/AIA in the 94 and $131 \AA$ channels. Wang et al. (2015) reported the first AIA event revealing signatures in agreement with a fundamental standing slow-mode wave, and found quantitative evidence for thermal conduction suppression from the temperature and density perturbations in the hot loop plasma of $\gtrsim 9 \mathrm{MK}$. The present study extends the work of Wang et al. (2015) by using an alternative approach. We determine the polytropic index directly based on the polytropic assumption instead of invoking the linear approximation. The same results are obtained as in the linear approximation, indicating that the nonlinearity effect is negligible. We find that the flare loop cools slower (by a factor of 2-4) than expected from the classical Spitzer conductive cooling, approximately consistent with the result of conduction suppression obtained from the wave analysis. The modified Spitzer cooling timescales based on the nonlocal conduction approximation are consistent with the observed, suggesting that nonlocal conduction may account for the observed conduction suppression in this event. In addition, the conduction suppression mechanism predicts that larger flares may tend to be hotter than expected by the EM-T relation derived by Shibata \& Yokoyama (2002).
\end{abstract}

Keywords. Sun: Flares — Sun: corona - Sun: oscillations — waves — Sun: UV radiation

\section{Introduction}

The magnetically dominated plasma of the solar corona can support propagation of various types of magnetohydrodynamics (MHD) waves. Observations of these waves allow us to determine physical parameters of coronal structures that cannot be measured directly via a technique called coronal seismology (Roberts et al. 1983; Nakariakov \& Verwichte 2005; Liu \& Ofman 2014). The knowledge of the appropriate value of the polytropic index is important for understanding the energy transport and the relation between density, temperature and pressure in hydrodynamic and MHD models of the solar and stellar coronae as well as of space plasmas (e.g. Jacobs \& Poedts 2011). In contrast to the adiabatic index that is always $5 / 3$ for an ideal monatomic gas (or fully ionized ideal plasma), the polytropic index can have other values to account for the overall contribution of the different physical processes (e.g., heating, radiative cooling, and thermal conduction) in the energy equation. From Hinode/EIS observations of propagating slow magnetoacoustic waves in a coronal loop, Van Doorsselaere et al. (2011) estimated the polytropic index to be close to 1 , and suggested that thermal conduction is the dominant heat transport mechanism in the warm (1-2 MK) corona. 

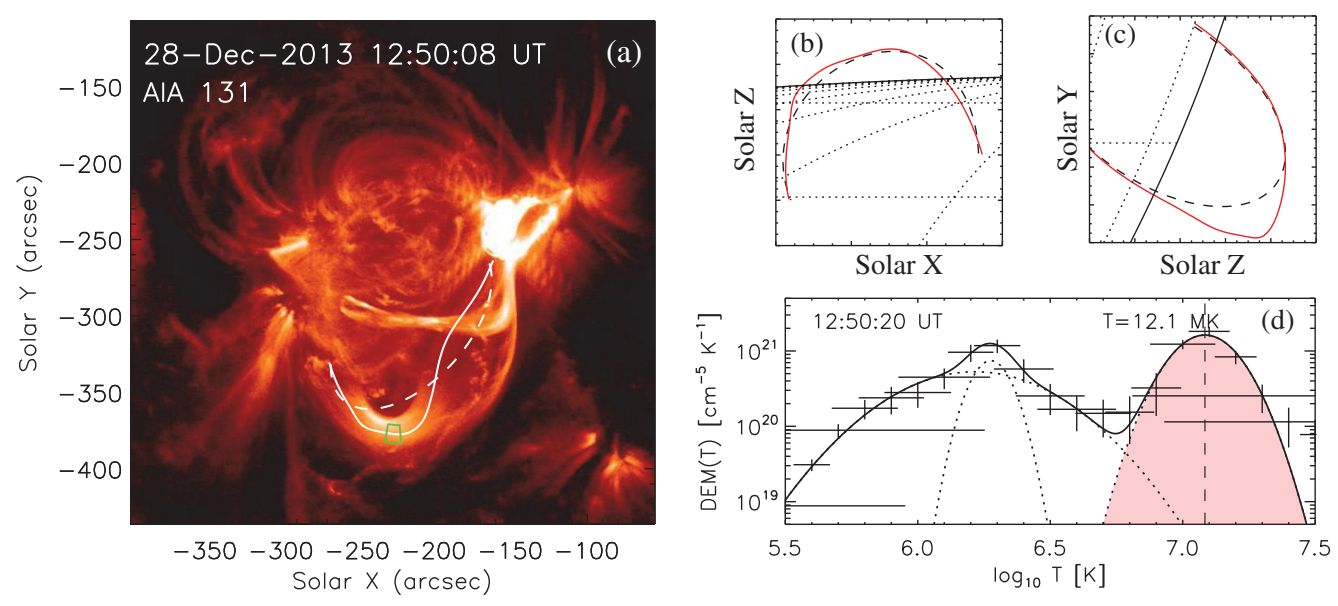

Figure 1. A longitudinal wave event observed by SDO/AIA on 2013 December 28. (a) $131 \AA$ image. The solid curve indicates the oscillating loop and its $3 \mathrm{D}$ reconstruction by the method of curvature radius maximization, and the dashed curve represents a fitted circular model. (b) and (c): The $2 \mathrm{D}$ projections of the loop models in the $\mathrm{XZ}$ and $\mathrm{YZ}$ planes, where $\mathrm{Z}$ is the direction of the observer's line-of-sight. (d) The derived differential emission measure (DEM) profile (crosses) for a small region $\left(9^{\prime \prime} \times 13^{\prime \prime}\right)$ marked with a box in (a). A fitted triple-Gaussian model (curves) is used to isolate the hot loop contribution (pink) from the background.

Impulsive energy release in a closed magnetic structure (so-called confined or noneruptive flares) provides a natural scenario for excitation of slow magnetoacoustic waves. This phenomenon was first discovered by the SOHO/SUMER spectrometer in hot coronal loops as Doppler shift oscillations in the flare lines (Wang et al. 2002; Wang 2011, for a review). These oscillations were mainly interpreted as fundamental standing slow modes because their phase speed is close to sound speed in the loop, and there is a quarter-period phase shift between the velocity and intensity oscillations in some events (Wang et al. 2003a,b). The initiation of the waves was often associated with small flares at the loop footpoint (Wang et al. 2005). These waves typically show a rapid decay. Theoretical and numerical studies suggested that the dominant dissipation mechanisms are thermal conduction (Ofman \& Wang 2002; De Moortel \& Hood 2003), compressive viscosity (Sigalotti et al. 2007), and nonlinearity effect (Ruderman 2013). These hot loop oscillation events are characterized by impulsive heating followed by a gradual cooling phase with similar features as solar flares, so also referred to as hot loop transient events (HLTEs, Curdt et al. 2004). The HLTEs observed in multiple spectral lines formed at different temperatures have been used to diagnose the heating function by a forward modeling approach (Taroyan et al. 2007). In addition, the wave periods were also used to determine the loop magnetic field by coronal seismology (Wang et al. 2007).

Recently, Kumar et al. (2013, 2015) reported longitudinal wave events observed with the Solar Dynamics Observatory (SDO)/Atmospheric Imaging Assembly (AIA). The waves have similar physical properties as hot loop oscillations observed by SUMER (Wang et al. 2003b, 2007), however, they bore the feature of bouncing back and forth in the heated loop, suggestive of a propagating wave. Wang et al. (2015) found the first AIA wave event in agreement with a fundamental standing slow mode wave, and clear evidence for thermal conduction suppression in the hot ( $\gtrsim 9 \mathrm{MK})$ flare loop by coronal seismology. They suggested that compressive viscosity dominates in the wave dissipation.

It is known that the classical Spitzer form of conductivity is valid under the assumptions that the electron velocity distribution is locally close to Maxwellian and the mean 

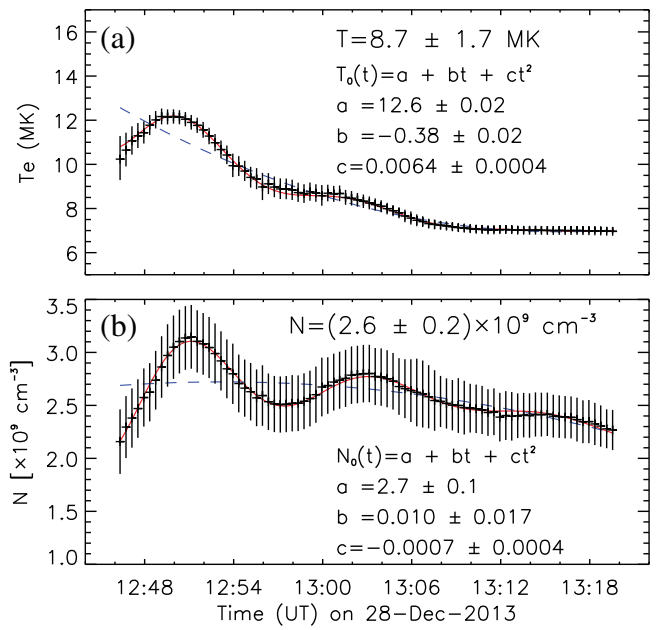
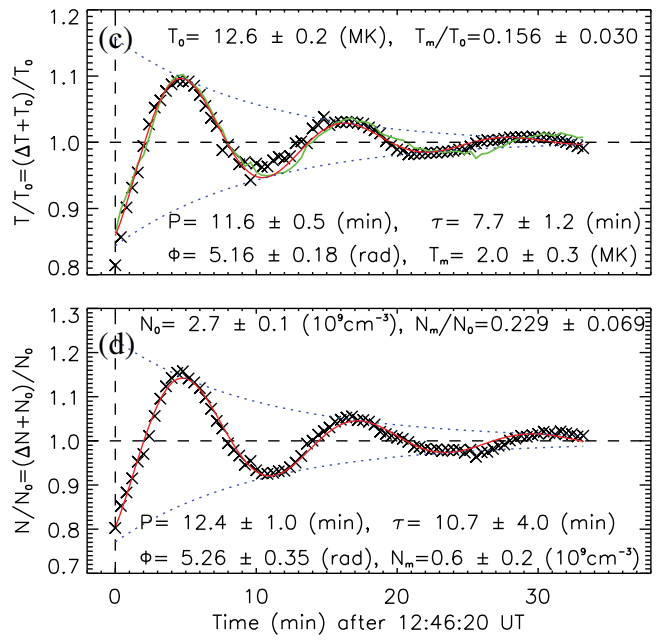

Figure 2. Evolution of the average temperature (a) and electron density (b) for the small loop segment shown in Figure 1(a). The solid curves show the best fit to the oscillatory signals, and the dashed curve is the parabolic trend. The error bars are the $1 \sigma$ error for the Gaussian fits of DEM. $T_{0}(t)$ and $N_{0}(t)$ marked on the plots are the fitted background trends for loop temperature and density. (c) Time profile of the temperature (crosses) and its best fit (thin solid curve) normalized to the background trend. (d) Same as (c) but for electron density. The measured physical parameters of the waves are marked on the plots. The thick solid curve in (c) is the expected variation in temperature derived from the observed density variation $N / N_{0}(t)$ for an adiabatic process.

free path $\lambda$ is much smaller than the temperature gradient scale length $L_{T}$ (Rosner et al. 1986). Laboratory experiments and numerical studies showed that the actual conductivity is smaller (by at least a factor of two) than that given by Spitzer when $L_{T} \lesssim 30 \lambda$ (e.g. Luciani et al. 1983). This is the case for typical solar flare loops with higher temperature $(T)$ because $\lambda$ increases with $T^{2}$. For example, for hot $(T=10 \mathrm{MK})$ loops with the length $L=10 \mathrm{Mm}$ and electron number density $n=10^{10} \mathrm{~cm}^{-3}$ (or $L=100$ $\mathrm{Mm}$ and $\left.n=10^{9} \mathrm{~cm}^{-3}\right)$, we find $L_{T} / \lambda \approx 7(L / 10 \mathrm{Mm})\left(n / 10^{10} \mathrm{~cm}^{-3}\right) /(T / 10 \mathrm{MK})^{2}<30$. For a long-duration event (LDE) with the slower-than-expected decay rate in soft X-ray (SXR), McTiernan et al. (1993) suggested that the long duration was caused by either continuous heating (after the impulsive burst in hard X-ray) or thermal conduction suppression. By studying the evolution of the SXR loop-top sources, Jiang et al. (2006) showed that plasma waves or turbulence may play an important role in suppressing the conduction during the decay phase of flares.

The study presented here is a supplement to Wang et al. (2015) (thereafter, called Paper I). We determine the polytropic index directly based on the polytropic assumption to examine the possible effect of nonlinearity on measurements. We also explore the effect of conduction suppression on the flare loop cooling, and discuss the possible cause.

\section{Observations and Results}

We analyzed the loop dynamics and thermal property using the SDO/AIA data. Figure 1(a) shows that a longitudinal wave event was triggered in a large hot loop by a C-class flare near the footpoint seen in the $131 \AA$ channel $(\sim 11 \mathrm{MK})$. The loop oscillations displayed as alternate brightenings in the two opposite legs, which can be obviously seen in a time-distance plot and the animations in Paper I. The oscillation period $(P)$ is 

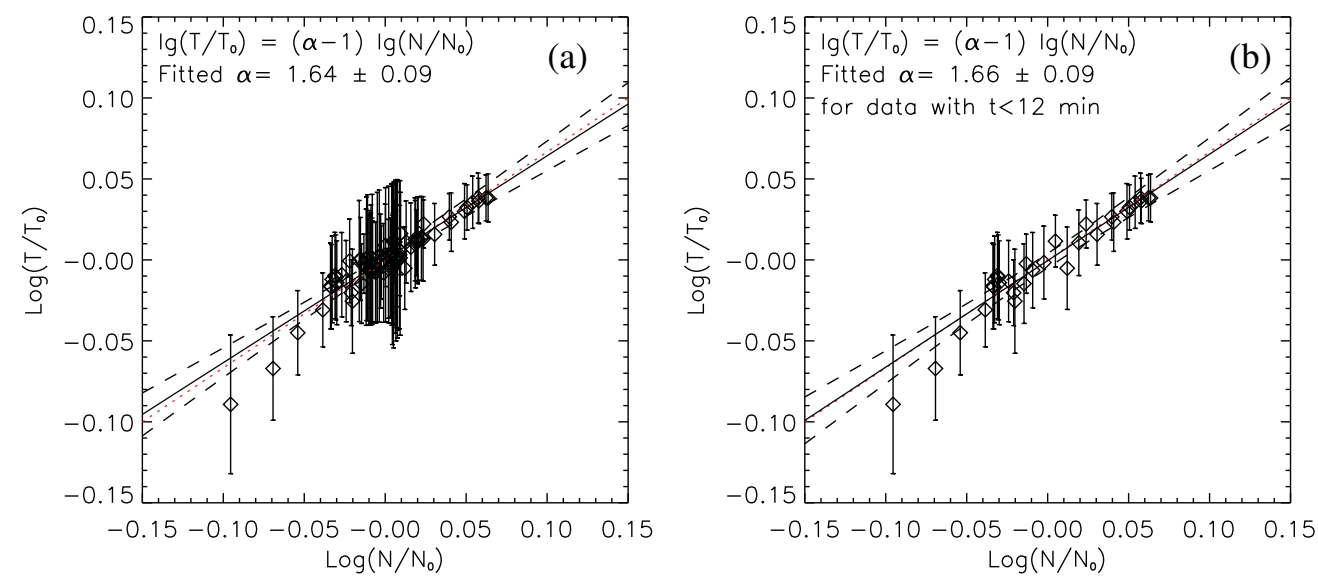

Figure 3. Measurements of the polytropic index $(\alpha)$. (a) The scatter plot of the electron density and temperature variations normalized to the background trend (diamonds) in logarithm, with the linear fit (solid line) and the line of $\gamma=5 / 3$ (dotted). The dashed lines indicate the $\pm 1 \sigma$ fitting error. (b) Same as (a) but for the data during the initial 12-min time. The measured values of $\alpha$ are marked on the plots.

about $12 \mathrm{~min}$. The loop length $(L)$ is an important parameter for identifying the wave mode. We determine the loop 3D geometry using the curvature radius maximization method which assumes the line-of-sight (LOS) coordinates $(\mathrm{Z})$ of the observed loop to be same as those of a circular model (Aschwanden 2009). Figure 1 shows the solution of the $3 \mathrm{D}$ reconstruction (which has an identical 2D projection as the observed loop), with de-projections into the XZ- and YZ-planes. We obtained the loop length $L \simeq 180 \mathrm{Mm}$, and an estimate of the wave phase speed $V_{p}=2 L / P \simeq 500 \mathrm{~km} \mathrm{~s}^{-1}$. The phase speed is close to the sound speed of $480 \mathrm{~km} \mathrm{~s}^{-1}$ for the hot loop of $\sim 10 \mathrm{MK}$ supporting the interpretation of the observed waves as a fundamental standing slow mode.

We utilized a regularized differential emission measure (DEM) analysis on AIA images in six extreme-ultraviolet (EUV) bands to diagnose the temperature and electron density of the oscillating loop (Hannah \& Kontar 2013). In inversions we took a $10 \%$ uncertainty for the $94 \AA$ and $131 \AA$ channels while a $20 \%$ uncertainty for the other channels because the oscillations were mainly seen in the hot channels. Figure $1(\mathrm{~d})$ shows the derived DEM profile for a small segment located at the brightest part of the loop. By assuming that the hottest component in triple-Gaussian fits came from the flare loop, we determined the temperature and electron density of the oscillating loop (see the details in Paper I and Sun et al. 2013). Figures 2(a) and (b) show their temporal variations. By fitting to a damped sine-function with a parabolic trend, we measured the physical parameters of the wave and loop background plasma which are marked on the plots.

We found that the temperature and density oscillations have similar periods and they are nearly in phase (Figs. 2(c) and (d)). The phase shift measured using the cross correlation is about $12^{\circ}$ which corresponds to the data cadence of $24 \mathrm{~s}$. This nearly inphase relationship suggests an adiabatic process on the timescale of oscillations because otherwise a large phase shift between the temperature and density oscillations caused by the nonideal effects such as conductive loss at higher temperature plasma would be expected (see Paper I). We calculated the expected variation in temperature $\left(T / T_{0}(t)\right)$ during an adiabatic process from the measured density variation $\left(N / N_{0}(t)\right)$ using $T / T_{0}=\left(N / N_{0}\right)^{\gamma-1}$ (assuming the adiabatic index $\gamma=5 / 3$ for fully ionized coronal plasma). We found that the expected and observed variations are in good agreement (except for the near-ending 

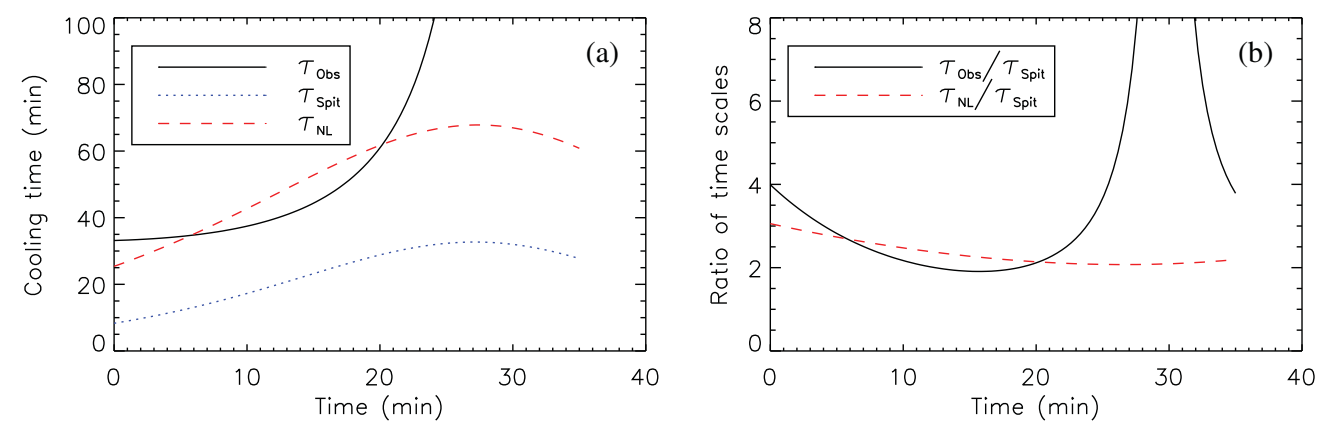

Figure 4. (a) The cooling times estimated from the observed temperature evolution (solid line), Spitzer conduction (dotted line), and nonlocal conduction approximation (dashed line). (b) The ratios of the measured cooling timescale to that of Spizter (solid line), and to that of nonlocal conduction (dashed line).

time of 10 min, see Fig. 2(c)). We quantitatively measured the polytropic index $\alpha$ under the polytropic assumption using the following linear relationship,

$$
\log \left(\frac{T}{T_{0}}\right)=(\alpha-1) \log \left(\frac{N}{N_{0}}\right) .
$$

This method is distinct from that used in Paper I where a linear approximation was made. Note that to correctly apply Eq. (2.1) to measure $\alpha$ the phase shift between $T / T_{0}$ and $N / N_{0}$ (if non-negligible) should be first removed. Figure 3 shows the linear-squares fitting for measurements of $\alpha$ in the two cases: $\alpha=1.64 \pm 0.09$ for all the data and $\alpha=1.66 \pm 0.09$ for only the data with $t<12 \mathrm{~min}$, where the uncertainty is the $1 \sigma$ error from the fit. We found that the measured values for $\alpha$ are same as those in Paper I. This indicates that the effect of nonlinearity is negligible, consistent with the signature that the temperature and density oscillations follow well the (damped) sinusoidal wave.

\section{Discussion and Conclusions}

We studied a longitudinal wave event triggered by the non-eruptive flare using SDO/ AIA. The waves in the hot flaring loop were identified as a fundamental standing slow mode. We analyzed the plasma thermal and wave properties of the oscillating loop, and found that its temperature and electron density variations are nearly in phase and the measured polytropic index $\alpha$ agrees well with the adiabatic index of $5 / 3$ for a fully ionized ideal plasma. These results imply that the MHD energy equation can be well represented with an adiabatic form, or the nonideal effects such as the stratification, optically thin radiative loss, and heat conduction are negligible during the oscillation period. In $\mathrm{Pa}-$ per I, based on a 1D linear MHD model of slow waves, we argued that because thermal conduction dominates in the energy equation in the hot $(\gtrsim 9 \mathrm{MK})$ plasma, the interpretation suggests a significant reduction of thermal conductivity (by at least a factor of 3 as estimated quantitatively).

The dissipation of slow waves by thermal conduction is due to temperature variations along the loop caused by the wave, while thermal conduction causes the hot loop cooling due to its rooting on the cool chromosphere. Now that thermal conduction is suppressed as known from the wave analysis, its influence on the loop cooling would also be expected. Figure 4 shows that the observed cooling timescales (calculated by $\tau_{\text {obs }}=T /(d T / d t)$ ) is about a factor 2-4 longer than the cooling timescales based on Spitzer's thermal conductivity $\left(\tau_{\text {Spit }}\right.$ using Eq. (D2) in Sun et al. 2013). This slower-than-expected cooling rate 
can be explained by conduction suppression which has a suppression factor consistent with that inferred from the wave analysis. For the mean temperature and density, we obtained $L_{T} / \lambda \sim 40$ where $L_{T}$ is taken as the loop length $L$. Under the nonlocal conduction approximation (Jiang et al. 2006), we calculated the modified Spitzer cooling timescales by $\tau_{\mathrm{NL}} \simeq 9.1\left(L_{T} / \lambda\right)^{-0.36} \tau_{\text {Spit }}$. Figure 4 shows a good agreement between $\tau_{\mathrm{NL}}$ and $\tau_{\text {obs }}$, suggesting that the nonlocal conduction in hot plasmas can account for the observed conduction suppression in this event.

In addition, the thermal conduction suppression mechanism may be used to explain the phenomenon that the larger (solar and stellar) flares tend to be hotter than expected by the EM- $T$ relation where $T$ is the peak temperature and EM the volume emission measure (Feldman et al. 1995; Shibata \& Yokoyama 2002). Assuming the balance between conduction cooling and reconnection heating and the pressure balance for flare loops, Shibata \& Yokoyama (2002) derived the scaling law EM $\propto B^{-5} T^{17 / 2}$ where $B$ is the magnetic field strength. If considering the suppressed conductivity $\kappa_{S}=\kappa_{0} / S$ where $\kappa_{0} \simeq 10^{-6} \mathrm{cgs}$ is the classical Spitzer conductivity and $S$ the suppression factor, we obtained the modified scaling law $\mathrm{EM}_{S} \propto S^{-3} B^{-5} T^{17 / 2}$ as well as the relations $T_{S} / T=S^{6 / 17}$ and $B_{S} / B=1 / S^{3 / 5}$. Given $S=3$, for instance, we estimated that the conduction suppression would lead to the flare loop hotter by about a factor 1.5 ; if the conduction suppression effect is neglected, the magnetic field strength of stellar flares may be overestimated by a factor of 2 from fitting the observed EM- $T$ diagram.

The work of TW and LO was supported by NASA grant NNX12AB34G and the NASA Cooperative Agreement NNG11PL10A to CUA. EP thanks the support from NASA grant NNX12AB34G. SDO is a mission for NASA's Living With a Star (LWS) program.

\section{References}

Aschwanden, M. J. 2009, Space Sci. Rev., 149, 31

Curdt, W., Wang, T. J., \& Dwivedi, B. N., et al. 2004, in: H. Lacoste (ed.), Proc. of SOHO 13 - Waves, Oscillations and Small-Scale Transient Events, ESA SP-547, p. 333

De Moortel, I. \& Hood, A. W. 2003, A\&广A, 408, 755

Feldman, U., Laming, J. M., \& Doschek, G. A. 1995, ApJ, 451, L79

Hannah, I. G. \& Kontar, E. P. 2013, A\&A, 553, A10

Jacobs, C. \& Poedts, S. 2011, Adv. Space Res., 48, 1958

Jiang, Y., Liu, S., Liu, W., \& Petrosian, V. 2006, ApJ, 638, 1140

Kumar, P. , Innes, D. E., \& Inhester, B. 2013, ApJ, 779, L7

Kumar, P. Kumar, P., Nakariakov, V. M., \& Cho, K.-S., ApJ, 804, 4

Liu, W. \& Ofman, L. 2014, Solar Phys., 289, 3233

Nakariakov, V. M. \& Verwichte, E. 2005, Living Rev. in Sol. Phys., 2, 3

Luciani, J. F., Mora, P., \& Virmont, J. 1983, Phys. Rev. Lett., 51, 1664

McTiernan, J. M., Kane, S. R., \& Loran, J. M., et al. 1993, ApJ, 416, L91

Ofman, L. \& Wang, T. J. 2002, ApJ, 580, L85

Shibata, K. \& Yokoyama, T. 2002, ApJ, 577, 422

Roberts, B., Edwin, P. M., \& Benz, A. O. 1983, Nature, 305, 688

Rosner, R., Low, B. C., \& Holzer, T. E. 1986, in: P. A. Sturrock (ed.), Physics of the Sun. II (Dordrecht: Reidel), p. 135

Ruderman, M. S. 2013, A\& $A, 553$, A23

Sigalotti, L. Di G., Mendoza-Briceño, C. A., \& Luna-Cardozo, M. 2007, Solar Phys., 246, 187

Sun, X., Hoeksema, J. T., \& Liu, Y., et al. 2013, ApJ, 778, 139

Taroyan, Y., Erdélyi, R., Wang, T. J., \& Bradshaw, S. J. 2007, ApJ, 659, L173

Van Doorsselaere, T., Wardle, N., Del Zanna, G., et al. 2011, ApJ, 727, L32

Wang, T. J. 2011, Space Sci. Rev., 158, 397 
Wang, T., Solanki, S. K., Curdt, W., Innes, D. E., \& Dammasch, I. E. 2002, ApJ, 574, L101

Wang, T. J., Solanki, S. K., Innes, D. E., Curdt, W., \& Marsch, E. 2003a, A $\& A$, 402, L17

Wang, T. J., Solanki, S. K., Curdt, W., et al. 2003b, A\& $A$, 406, 1105

Wang, T. J., Solanki, S. K., Innes, D. E., \& Curdt, W. 2005, A\&3A, 435, 753

Wang, T. J., Innes, D. E., \& Qiu, J. 2007, ApJ, 656, 598

Wang, T. J., Ofman, L., Sun, X., Provornikova, E., \& Davila, J. M. 2015, ApJ, 811, L13 (Paper I) 